\title{
Comparison of the safety and efficacy of the fixed combination of dorzolamide/timolol and the concomitant administration of dorzolamide and timolol: a clinical equivalence study
}

\author{
Jill Hutzelmann, Susan Owens, Arthur Shedden, Ingrid Adamsons, Enrique Vargas, and
} the International Clinical Equivalence Study Group

\begin{abstract}
Aims-To compare the tolerability and efficacy of a fixed combination solution of dorzolamide/timolol (Cosopt), administered twice daily with the concomitant administration of its components, dorzolamide (Trusopt) twice daily and timolol (Timoptic) twice daily.

Methods-After a 2 week timolol run in, patients with open angle glaucoma or ocular hypertension were randomised $(1: 1)$ to receive treatment with either the dorzolamide/timolol combination solution twice daily (combination) or the dorzolamide solution twice daily plus timolol maleate solution twice daily (concomitant) for 3 months.

Results-299 patients were entered and 290 patients completed the study. Compared with the timolol baseline, additional IOP lowering of $16 \%$ was observed at trough (hour 0 ) and $22 \%$ at peak (hour 2) at month 3 in both the concomitant and combination groups. The IOP lowering effects of the two treatment groups were clinically and statistically equivalent as demonstrated by the extremely small point differences (concomitant - combination) observed in this study $-0.01 \mathrm{~mm} \mathrm{Hg}$ at trough and 0.08 $\mathrm{mm} \mathrm{Hg}$ at peak. The safety variables of the concomitant and combination groups were very similar. Both combination and concomitant therapy were well tolerated and few patients discontinued due to adverse effects.

Conclusions-The dorzolamide/timolol combination solution administered twice daily is equivalent in efficacy and has a similar safety profile to the concomitant administration of the components administered twice daily.

(Br f Ophthalmol 1998;82:1249-1253)
\end{abstract}

S Owens

A Shedden

I Adamsons

National Institute of Ophthalmology, Lima, Peru

E Vargas

Correspondence to:

J Hutzelmann, Merck \& Co,

Inc, Clinical Research, BL

1-3, 10 Sentry Parkway, Blue Bell, PA 19422, USA.

Accepted for publication 15 April 1998 is a chronic progressive disease, the majority of patients eventually require additional medi- cation for control of IOP. In previous clinical studies, dorzolamide demonstrated a clinically significant additive effect ranging from a $13-21 \%$ further reduction in IOP when added to ophthalmic $\beta$ blockers. ${ }^{2}{ }^{3}$ This additive effect supported the development of a combination solution of dorzolamide and timolol (Cosopt), which is administered twice daily. Other agents additive to timolol (such as pilocarpine, adrenaline (epinephrine), and oral carbonic anhydrase inhibitors) have side effects which may often result in discontinuation. However, the discontinuation rate due to side effects has been low with dorzolamide and timolol. ${ }^{45}$ In a crossover study, patients preferred therapy with dorzolamide and timolol to therapy with pilocarpine and timolol by more than $7: 1 .^{4}$ This combination product may also improve patient compliance with therapy since compliance decreases as the dosing frequency increases. Thus, a twice daily combination solution should lead to improved compliance compared with concomitant therapy with timolol and either dorzolamide, or other alternative agents which may have dosing regimens of up to four times daily.

The safety and efficacy of this combination solution has been evaluated in several large phase III studies in patients with ocular hypertension or open angle glaucoma. One study compared the $2.0 \%$ dorzolamide $/ 0.5 \%$ timolol combination twice daily with the concomitant administration of $0.5 \%$ timolol twice daily and $2.0 \%$ dorzolamide three times daily (the US dosage regimen for "add on" therapy in patients with ocular hypertension or open angle glaucoma). That study showed that the IOP lowering effect of the combination was equivalent $(\geqslant 95 \%$ confidence that the absolute difference between treatments in mean IOP change from baseline was $\leqslant 1.5 \mathrm{~mm} \mathrm{Hg}$ ) to that of the concomitant treatment at hours 0 , 2 , and 8 across the entire 3 month treatment period, although the point estimates at trough slightly favoured the concomitant treatment group (by $<1 \mathrm{~mm} \mathrm{Hg}$ ). ${ }^{6}$ In a study comparing the $2.0 \%$ dorzolamide $/ 0.5 \%$ timolol combination twice daily with monotherapy with either $2.0 \%$ dorzolamide three times daily or $0.5 \%$ timolol twice daily, the IOP lowering of the combination was $27 \%$ at trough and $33 \%$ at peak from the untreated baseline. ${ }^{7}$

These previous clinical studies compared the combination with the components administered as monotherapy or administered 
concomitantly in their usual monotherapy regimens - that is, twice daily dosing for timolol and three times daily dosing for dorzolamide. However, no clinical study had yet compared the components administered concomitantly in the same dosing regimen as is recommended in most countries for add on therapy - that is, twice daily dosing for both timolol and dorzolamide. This study was performed to determine whether the $2.0 \%$ dorzolamide $/ 0.5 \%$ timolol combination twice daily is equivalent in lowering IOP compared with the components administered twice daily in patients with bilateral ocular hypertension or open angle glaucoma whose IOP is not adequately controlled with timolol twice daily alone.

\section{Materials and methods \\ STUDY DESIGN}

This study was a parallel, randomised, double masked, active controlled, multicentre study conducted at 16 sites throughout the world (the names of the investigators and their sites are given in the acknowledgments). Male and female patients, 21-85 years of age, with a diagnosis of either ocular hypertension or open angle glaucoma in both eyes were eligible to participate. Patients were excluded from the study for reasons which included ocular surgery within 6 months ( 3 months for laser) of study start; history or evidence of bronchial asthma, clinically significant chronic obstructive pulmonary disease, or impaired renal function; or a contraindication to dorzolamide or timolol. Eligible patients discontinued any previous ocular hypotensive therapy and began a 2 week run in on $0.5 \%$ timolol twice daily. During this period, a complete ophthalmic examination was performed. To monitor compliance during the timolol run in, patients returned on day -7 for an IOP measurement. Patients returned again at the end of the 2 week timolol run in (day 1), for a baseline evaluation. If the patient's IOP was $\geqslant 22 \mathrm{~mm} \mathrm{Hg}$ in one or both eyes at the trough measurement (hour $0 ; 0830$ ) and peak (hour 2;1030), then the patient was randomised $1: 1$ to receive either the $2.0 \%$ dorzolamide $/ 0.5 \%$ timolol combination solution twice daily (combination group) or concomitant treatment with $2 \%$ dorzolamide twice daily and $0.5 \%$ timolol twice daily (concomitant group). The combination group also received a placebo solution twice daily. Therefore each patient received two study drug components to keep the study groups masked. The study drugs were colour coded to distinguish between the two components that each patient received. The study drug was instilled at morning and bedtime. The timolol or combination solution was administered first, followed 10 minutes later by administration of the dorzolamide or placebo solution.

Patients returned to the clinic on days 15 , 30,60 , and 90. At all study visits, an evaluation of symptoms and an external and anterior segment examination were performed at hour 0 and 2; visual acuity was measured at hour 0 only. Within 5 days of completing or discon- tinuing the study, each patient had a dilated ophthalmoscopy, and a visual field examination. If these examinations were performed on the final study day, then mydriatic agents were not instilled until after the final IOP measurement was taken.

EVALUATION CRITERIA

IOP was measured at hours 0 and 2 on day 1 (baseline) and on days 15,30,60, and 90. The primary efficacy variable was the mean change in IOP from the time matched baseline value in the worse eye. Safety was evaluated by monitoring patients for clinical adverse experiences, ocular signs and symptoms, and changes in visual acuity, visual field, and cup/disc ratio.

\section{ANALYTICAL METHODS}

Ocular hypotensive effect was assessed using absolute change in IOP from the time matched baseline values (trough and peak) using the patient's worse eye. The two treatment groups were compared to determine whether they are equivalent in their ability to reduce IOP. Treatment group equivalency was assessed with the calculation of the $90 \%$ confidence level that the means of the two treatment groups differ by $\leqslant 1.5 \mathrm{~mm} \mathrm{Hg}$. Treatment equivalence was based on an average of the observed month 2 and month 3 data.

Treatment group comparisons with regard to adverse experiences, ocular signs and symptoms, and incidence of visual field defects were made using Fisher's exact test (two tailed). All $\mathrm{p}$ values were rounded to three decimal places, and statistical significance was declared if the rounded $\mathrm{p}$ value was less than or equal to 0.050 .

\section{Results}

A total of 299 patients were entered into the study; 151 patients received $2.0 \%$ dorzolamide $/ 0.5 \%$ timolol combination solution (twice daily) and 148 received concomitant administration of $2.0 \%$ dorzolamide solution (twice daily) and $0.5 \%$ timolol solution (twice daily). The demographics of patients in both treatment groups were similar. Approximately two thirds of patients were female and one third were male. The majority of patients were white (52\%), 23\% were Mestizo, 19\% were Hispanic, and 5\% were black. Characteristics of iris colour, age, and baseline IOP (worse eye) were also evenly distributed between the two treatment groups (Table 1).

Of the 299 patients entered, 290 (97\%) completed the study. Of the nine patients who discontinued early, three discontinued due to clinical adverse experiences (two in the combination group and one in the concomitant group). Of the remaining six patients, four discontinued owing to insufficient IOP control (three in the combination group and one in the concomitant group), one patient was lost to follow up, and one patient discontinued due to a protocol violation.

\section{EFFICACY}

Changes in IOP from the timolol baseline ranged from $-3.8 \mathrm{~mm} \mathrm{Hg}$ to $-5.8 \mathrm{~mm} \mathrm{Hg}$ for 
Table 1 Baseline demographic characteristics by treatment $\operatorname{group}^{\star}$

\begin{tabular}{|c|c|c|c|}
\hline & $\begin{array}{l}\text { Combination } \\
(n=151)\end{array}$ & $\begin{array}{l}\text { Concomitant } \\
(n=148)\end{array}$ & $\begin{array}{l}\text { Total } \\
(n=299)\end{array}$ \\
\hline & No $(\%)$ & No $(\%)$ & No $(\%)$ \\
\hline \multicolumn{4}{|l|}{ Sex: } \\
\hline Male & $65(43)$ & $48(32)$ & $113(38)$ \\
\hline Female & $86(57)$ & $100(68)$ & $186(62)$ \\
\hline \multicolumn{4}{|l|}{ Race: } \\
\hline White & $79(52)$ & $76(51)$ & $155(52)$ \\
\hline Black & $7(5)$ & $8(5)$ & $15(5)$ \\
\hline Hispanic & $29(19)$ & $28(19)$ & $57(19)$ \\
\hline Mestizo & $33(22)$ & $35(24)$ & $68(23)$ \\
\hline Other & $3(2)$ & $1(1)$ & $4(1)$ \\
\hline \multicolumn{4}{|l|}{ Iris colour: } \\
\hline Dark & $113(75)$ & $109(74)$ & $222(74)$ \\
\hline Light & $38(25)$ & $39(26)$ & $77(26)$ \\
\hline \multicolumn{4}{|l|}{ Age: } \\
\hline Mean (SD) & $62.6(11.4)$ & $63.6(10.6)$ & $63.1(11.0)$ \\
\hline Range & $23-83$ & $28-84$ & $23-84$ \\
\hline \multicolumn{4}{|c|}{ Baseline IOP (mm Hg) worse eye: } \\
\hline \multicolumn{4}{|c|}{ Hour 0 (trough) } \\
\hline Mean (SD) & $25.6(3.1)$ & $25.3(3.2)$ & $25.4(3.2)$ \\
\hline Range & $22-37$ & $19-36$ & $19-37$ \\
\hline \multicolumn{4}{|l|}{ Hour 2 (peak) } \\
\hline Mean (SD) & $24.7(3.2)$ & $24.5(3.2)$ & $24.6(3.2)$ \\
\hline Range & $20-38$ & $16-37$ & $16-38$ \\
\hline
\end{tabular}

^No significant differences between treatment groups were found.

the combination group and from $-3.6 \mathrm{~mm} \mathrm{Hg}$ to $-5.8 \mathrm{~mm} \mathrm{Hg}$ for the concomitant group over the week 2 , months 1,2 , and 3 visits (Table 2). The correlating percentage changes in IOP from baseline ranged from $-14.6 \%$ to $-23.3 \%$ for the combination group and $-14.0 \%$ to $-23.2 \%$ for the concomitant group. At the trough time point (hour 0), the IOP effect for the combination group ranged from -3.8 to $-4.5 \mathrm{~mm} \mathrm{Hg}(-14.6 \%$ to $-17.2 \%)$, while the concomitant group had similar IOP

Table 2 IOP summary statistics * $(\mathrm{mm} \mathrm{Hg})$

\begin{tabular}{|c|c|c|c|c|c|c|}
\hline \multirow[b]{2}{*}{ Exam } & \multirow[b]{2}{*}{ Treatment } & \multirow[b]{2}{*}{ No } & \multirow{2}{*}{$\frac{\text { Baseline }}{\text { Mean (SD) }}$} & \multirow{2}{*}{$\frac{\text { Treatment }}{\text { Mean }(S D)}$} & \multirow{2}{*}{$\frac{\text { Change }}{\text { Mean (SD) }}$} & \multirow{2}{*}{$\frac{\% \text { Change }}{\text { Mean (SD) }}$} \\
\hline & & & & & & \\
\hline \multicolumn{7}{|l|}{ Hour 0: } \\
\hline \multirow[t]{2}{*}{ Week 2} & Combination & 150 & $25.6(3.1)$ & $21.8(4.0)$ & $-3.8(3.2)$ & $-14.6(11.9)$ \\
\hline & Concomitant & 147 & $25.3(3.2)$ & $21.7(3.8)$ & $-3.6(3.3)$ & $-14.0(12.8)$ \\
\hline \multirow[t]{2}{*}{ Month 1} & Combination & 151 & $25.6(3.1)$ & $21.1(3.9)$ & $-4.5(3.3)$ & $-17.2(12.5)$ \\
\hline & Concomitant & 148 & $25.3(3.2)$ & $21.0(3.8)$ & $-4.3(3.3)$ & $-16.9(12.5)$ \\
\hline \multirow[t]{2}{*}{ Month 2} & Combination & 151 & $25.6(3.1)$ & $21.4(4.1)$ & $-4.1(3.3)$ & $-16.1(12.3)$ \\
\hline & Concomitant & 148 & $25.3(3.2)$ & $21.0(4.0)$ & $-4.3(3.3)$ & $-17.0(12.4)$ \\
\hline \multirow[t]{2}{*}{ Month 3} & Combination & 151 & $25.6(3.1)$ & $21.4(4.1)$ & $-4.2(3.3)$ & $-16.3(12.5)$ \\
\hline & Concomitant & 148 & $25.3(3.2)$ & $21.1(3.7)$ & $-4.2(3.1)$ & $-16.3(11.5)$ \\
\hline \multicolumn{7}{|l|}{ Hour 2: } \\
\hline \multirow[t]{2}{*}{ Week 2} & Combination & 151 & $24.7(3.2)$ & $19.5(3.6)$ & $-5.2(2.9)$ & $-20.9(11.1)$ \\
\hline & Concomitant & 148 & $24.5(3.2)$ & $19.4(3.5)$ & $-5.1(3.3)$ & $-20.6(12.4)$ \\
\hline \multirow[t]{2}{*}{ Month 1} & Combination & 151 & $24.7(3.2)$ & $19.0(3.9)$ & $-5.7(3.2)$ & $-23.1(12.6)$ \\
\hline & Concomitant & 148 & $24.5(3.2)$ & $19.1(3.5)$ & $-5.4(3.5)$ & $-21.7(12.9)$ \\
\hline \multirow[t]{2}{*}{ Month 2} & Combination & 151 & $24.7(3.2)$ & $18.9(3.6)$ & $-5.8(3.0)$ & $-23.3(11.5)$ \\
\hline & Concomitant & 148 & $24.5(3.2)$ & $18.7(3.6)$ & $-5.8(3.4)$ & $-23.2(12.8)$ \\
\hline \multirow[t]{2}{*}{ Month 3} & Combination & 151 & $24.7(3.2)$ & $19.4(3.7)$ & $-5.4(3.1)$ & $-21.6(12.3)$ \\
\hline & Concomitant & 148 & $24.5(3.2)$ & $19.1(3.5)$ & $-5.4(3.3)$ & $-21.8(11.9)$ \\
\hline
\end{tabular}

* “All patients treated” analysis (last observation carried forward), worse eye.

Table 3 IOP estimates and confidence levels for the difference between treatments ( $m m \mathrm{Hg}$ ), mean change in IOP from baseline averaged over the month 2 and month 3 visits*

\begin{tabular}{lllll}
\hline Time point & $\begin{array}{c}\text { Difference between } \\
\text { treatments }\end{array}$ & $\begin{array}{l}\text { Standard error of } \\
\text { difference }\end{array}$ & $\begin{array}{l}90 \% \text { CI for difference } \\
\text { between means }\end{array}$ & $\begin{array}{l}\text { Confidence difference lies } \\
\text { between }-1.5 \text { and } 1.5\end{array}$ \\
\hline Hour 0 & 0.01 & 0.32 & $(-0.52,0.55)$ & $>0.999 \dagger$ \\
Hour 2 & 0.08 & 0.32 & $(-0.45,0.60)$ & $>0.999 \dagger$
\end{tabular}

* "All patients treated" analysis (observed cases), worse eye. Sample size (at hour 0 and 2): Concomitant, $\mathrm{n}=147$; Combination, $\mathrm{n}=145$.

The difference between treatments (concomitant - combination) is a weighted average of the mean difference within each clinic based on the number of patients enrolled at each clinic.

†The confidence is 0.900 or more that the difference between treatment group means lies between

$-1.5 \mathrm{~mm} \mathrm{Hg}$ and $1.5 \mathrm{~mm} \mathrm{Hg}$.

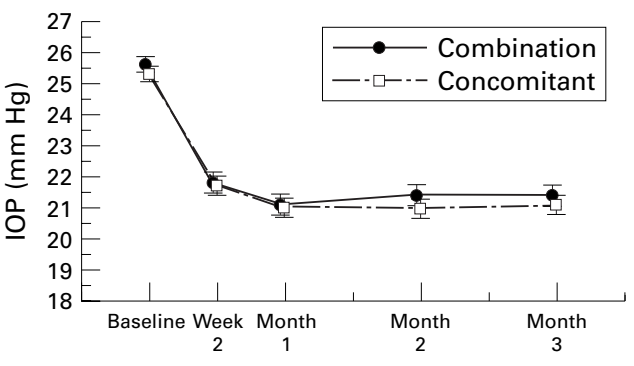

Figure 1 IOP treatment means and standard errors at hour 0 (trough) by study visit; "all patients treated" analysis (last observation carried forward), worse eye.

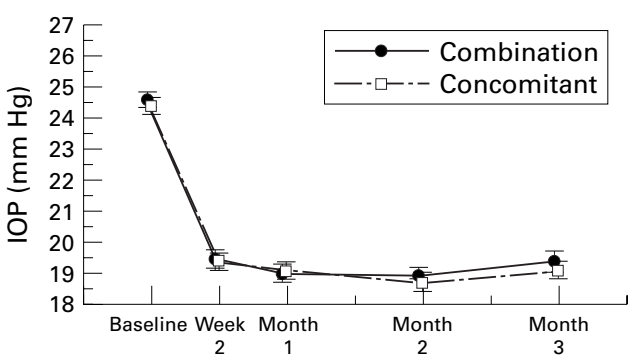

Figure 2 IOP treatment means and standard errors at hour 2 (peak) by study visit; "all patients treated analysis" (last observation carried forward), worse eye.

lowering ranging from $-3.6 \mathrm{~mm} \mathrm{Hg}$ to -4.3 $\mathrm{mm} \mathrm{Hg}(-14.0 \%$ to $-17.0 \%)$ (Fig 1$)$. The IOP lowering at peak (hour 2) ranged from -5.2 to $-5.8 \mathrm{~mm} \mathrm{Hg}(-20.9 \%$ to $-23.3 \%)$ for the combination group and -5.1 to $-5.8 \mathrm{~mm} \mathrm{Hg}$ $(-20.6 \%$ to $-23.2 \%)$ for the concomitant group (Fig 2). Mean reductions in IOP at hour 2 were roughly $1.3 \mathrm{~mm} \mathrm{Hg} \mathrm{(5.9 \% )} \mathrm{greater} \mathrm{than}$ reductions in IOP at hour 0 for each visit. At both trough and peak, the IOP continued to drop between the week 2 and month 1 time points; however, the reduction in IOP stabilised by month 1 and was essentially maintained through month 3 . The difference in mean IOP lowering from baseline between treatment groups (concomitant - combination) at months 2 and 3 (averaged) was 0.01 $\mathrm{mm} \mathrm{Hg}$ at trough (hour 0) and $0.08 \mathrm{~mm} \mathrm{Hg}$ at peak (hour 2). The positive difference indicates that the IOP reduction in the combination group was negligibly greater on average than the IOP reduction in the concomitant group. The $90 \%$ confidence intervals for the difference in treatment group means were $(-0.52$ $\mathrm{mm} \mathrm{Hg}, 0.55 \mathrm{~mm} \mathrm{Hg}$ ) at hour 0 and $(-0.45$, 0.60 ) at hour 2. Since zero was included in the confidence intervals at both time points it can be concluded that the treatments are equivalent $(\leqslant 1.5 \mathrm{~mm} \mathrm{Hg}$ in mean IOP change between treatment groups) in IOP lowering effect (Table 3).

The averaging of month 2 and month 3 time points was validated since there was $>99.9 \%$ confidence that the difference in mean change between the month 2 and 3 visits was within $-1.5 \mathrm{~mm} \mathrm{Hg}$ and $1.5 \mathrm{~mm} \mathrm{Hg}$. Baseline covariates of investigator, age $(<65, \geqslant 65)$, race (white, other), sex, and iris colour (dark, light) were also explored for an effect on change in IOP (average of month 2 and month 3) from baseline and for interaction with treatment. 
Overall, there was no evidence of a covariant by treatment interaction. Significant independent effects on mean change in IOP due to investigator and sex were observed; there was a greater reduction in IOP for males than for females. A significant independent effect due to race was also observed at the hour 0 time point only. No other covariates were significant.

SAFETY

Signs and symptoms

In both treatment groups, $34 \%$ of patients experienced one or more ocular symptoms. The ocular symptoms with the highest overall incidence were burning, itching, and stinging. Burning was reported by $13 \%$ of patients in the combination group compared with $9 \%$ of patients in the concomitant group. Stinging was reported by $9 \%$ of patients in each treatment group, and itching was reported by $9 \%$ of combination patients and $6 \%$ of concomitant patients. The non-ocular symptom of bitter taste was reported by $38 \%$ of patients in the combination group and $42 \%$ of patients in the concomitant group. There were no statistically significant differences between the treatment groups with regard to the incidence of this or any other non-ocular symptom. The only symptom that was statistically significant different between the treatment groups was eye pain; $4 \%$ in the concomitant group $v 0 \%$ in the combination group $(\mathrm{p}=0.014)$.

The most frequently reported ocular sign in this study was conjunctival hyperaemia ( $9 \%$ in both groups). Compared with patients in the combination group, patients in the concomitant group had a statistically greater incidence of all cornea signs $(14 \% v 5 \%, \mathrm{p}=0.011)$ and specifically for corneal superficial punctate keratitis (SPK), ( $7 \%$ v 1\%, $\mathrm{p}=0.005)$. The two treatment groups were similar with regard to visual acuity, visual field progression, or visual field defect; there were no statistically significant differences in the results of these evaluations between the two treatment groups. Also, no clinically meaningful changes in the visual field global indices were observed in either treatment group.

\section{Adverse experiences}

Emergent and worsening signs, symptoms, or conditions that were considered to be clinically significant were reported as adverse experiences. Seventy two patients $(48 \%)$ in the combination group and $76(51 \%)$ patients in the

Table 4 Clinical adverse experience summary

\begin{tabular}{|c|c|c|}
\hline & $\begin{array}{l}\text { Combination } \\
(n=151)\end{array}$ & $\begin{array}{l}\text { Concomitant } \\
(n=148)\end{array}$ \\
\hline & No $(\%)$ & No $(\%)$ \\
\hline Patients evaluated & 151 & 148 \\
\hline With any adverse experience & $72(48)$ & $76(51)$ \\
\hline Drug related adverse experience ${ }^{\star}$ & $36(24)$ & $43(29)$ \\
\hline Serious adverse experience & $1(1)$ & $0(0)$ \\
\hline Patients who died & $0(0)$ & $0(0)$ \\
\hline Discontinued owing to adverse experience & $2(1)$ & $1(1)$ \\
\hline
\end{tabular}

No significant differences between treatment groups were found.

*Drug related implies possibly, probably, or definitely caused by the test drug. concomitant group had one or more adverse experiences (Table 4). There were no statistically significant differences between the treatment groups in the proportion of patients with any adverse experience or with drug related adverse experiences. The most frequently reported adverse experiences were ocular burning and/or stinging (12\% in the combination group and $8 \%$ in the concomitant group) and taste perversion-for example, bitter taste (11\% in the combination group and $12 \%$ in the concomitant group). Investigators considered these two adverse experiences to be drug related in at least $80 \%$ of patients in both the concomitant and combination treatment groups.

\section{Discussion}

This study demonstrated that there was no clinical or statistical difference between the administration of $2.0 \%$ dorzolamide twice daily plus $0.5 \%$ timolol twice daily as a single combination product, or as separate individual products. At both trough (hour 0) and peak (hour 2), the point estimate for the treatment difference (concomitant - combination) was extremely small; $0.01 \mathrm{~mm} \mathrm{Hg}$ at trough and $0.08 \mathrm{~mm} \mathrm{Hg}$ at peak. In addition, there was $>99.9 \%$ confidence that the difference between treatment group means at both trough and peak were within $-1.5 \mathrm{~mm} \mathrm{Hg}$ and 1.5 $\mathrm{mm} \mathrm{Hg}$. Furthermore, this study demonstrated that patients on timolol achieve a substantial drop in IOP when they are switched to the combination therapy. This additional IOP lowering from the timolol baseline ranged from $-16 \%$ at trough to $-22 \%$ at peak after initiation of either combination or concomitant treatment. These results are similar to the add on effect observed in a previous 12 month study of the combination solution which found a $14 \%$ further decrease at trough and $21 \%$ decrease at peak, compared with the timolol baseline. ${ }^{6}$ The adjunctive effect of dorzolamide twice daily to timolol in this study also agrees with that observed in a previous study in which the IOP lowering of dorzolamide twice daily was similar to pilocarpine four times daily as add on therapy to timolol. ${ }^{3}$

The safety profile of the combination was also essentially equivalent to concomitant administration of its components. Both concomitant and combination therapy were well tolerated, and few patients discontinued for adverse effects. The rates of the most common symptoms of ocular burning, ocular stinging, and bitter taste were similar for the combination and concomitant treatments groups and agree with the incidence of these symptoms observed in previous studies with dorzolamide and with the dorzolamide/timolol combination. $^{67}$

In summary, this study has demonstrated that the $2.0 \%$ dorzolamide $/ 0.5 \%$ timolol combination administered twice daily provided additional IOP lowering in patients who were not adequately controlled on timolol alone. The combination solution was well tolerated and had equivalent efficacy and a similar safety profile to that of twice daily administration of 
$2.0 \%$ dorzolamide given concomitantly with twice daily administration of $0.5 \%$ timolol. The combination solution provides a dosing regimen which should improve compliance compared with concomitant products as well as products that are dosed three and four times daily, while offering equivalent efficacy and comparable tolerability.

Ms Hutzelmann, Ms Owens, Dr Shedden, and Dr Adamsons were employees of Merck \& Co, Inc, the manufacturer of timolol, dorzolamide, and the dorzolamide/timolol combination, at the time this study was conducted. The other author have no proprietary interest in timolol, dorzolamide, dorzolamide/timolol combination, or the trademark Merck.

Cosopt, Trusopt, and Timoptic are products of Merck \& Co, Inc, Whitehouse Station, NJ.

The authors would like to thank the investigators who participated in this study: Carlos Jiminez Antillon, Hospital Mexico, San Jose, Costa Rica; Eduardo Arenas, Private Mractice, San Jose, Costa Rica; Eduardo Arenas, Private General DeMexico SS, Mexico City, Mexico; Stephen Best, Auckland Hospital, Auckland, New Zealand; Anne Brooks, Auckland Hospital, Auckland, New Zealand; Anne Brooks, Royal Victorian Eye and Ear Hospital, Melbourne, Australia; Felix Gil-Carrasco, Hosp Dr Luis Sanchez Bulnes, Coyoacan, Mexico; Antonio Correa, Colombian Ophthalmological Assn, Bogata, Columbia; Andres Eggers, Del Salvador Hospital, Santiago, Chile; William E Gilles, Royal Victorian Eye and Ea Hospital, Melbourne, Australia; Newton Kara Jose, State University of Campinas, Sao Paulo, Brazil; Simon Fabian Lerner, Private Practice, Buenes Aires, Argentina; Moshe Lusky, Beilinson Medical Center, Petah, Tikva, Israel; Carlos
Portocarrero, Hospital Roosevelt, Guatemala, Guatemala; JeanPaul Renard, Val de Grace Army Hospital, Paris, France; George Theodosiadis, Kratiko Hospital, Athens, Greece; Joaquim Torres, Oporto University, Institute of Bio Medical Sciences, Oporto, Portugal; Enrique M Vargas, National Institute of Ophthamology, Lima, Peru.

1 Innovations in Ophthalmic Pharmaceuticals 1996-2005. Stanford, CA: Plan A, 1996:28-36.

2 Strahlman E, Tipping R, Vogel R, and the International Dorzolamide Study Group. A double-masked, randomized, one year study comparing dorzolamide, timolol and betaxolol. Arch Ophthalmol 1995;113:1009-16.

3 Strahlman E, Tipping R, Vogel R, et al and the Dorzolamide Additivity Study Group. The use of dorzolamide and pilocarpine as adjunctive therapy to timolol in patients with elevated intraocular pressure. Ophthalmology 1996;103: 1283-93.

4 Laibovitz R, Strahlman E, Barber B, et al. Comparison of quality of life and patient preference of dorzolamide and pilocarpine as adjunctive therapy to timolol in the treatment of glaucoma. F Glaucoma 1995;4:306-13.

5 Laibovitz R, Boyle J, Snyder E, et al. Dorzolamide versus pilocarpine as adjunctive therapy to timolol: a comparison of patient preference and impact on daily life. Clin Ther of patient prefer

6 Strohmaier K, Snyder E, Adamsons I. The long term safety and efficacy of COSOPT, a fixed combination of dorzolamide and timolol. Invest Ophthalmol Vis Sci 1996;37:S1102

7 Boyle J, Ghosh K, Gieser D, et al, and the Dorzolamide/ Timolol Study Group. A randomized trial comparing the dorzolamide/timolol combination given BID to monotherapy with timolol and dorzolamide. Ophthalmology (in press). 